\title{
Benchmark
}

\section{Direct Detection of Protein Thiol Derivatization by PAGE}

BioTechniques 33:1292-1295 (December 2002)

\section{Lela Johnson and Paul D. Gershon}

Texas A\&M University System Health Science Center, Houston, TX, USA

Derivatization of a protein thiol with a chemical reporter can be monitored directly for reporters possessing a suitable marker such as fluorescence or radioactivity. Thiol-reactive fluorescent markers might include thioglo (5), cpm $(6,8)$, or a thiol-reactive EuropiumEDTA chelate (3). Thiol-reactive radioactive markers might include ${ }^{125}$ I-labeled AET (2), or ${ }^{55} \mathrm{Fe}$-EDTA or ${ }^{63} \mathrm{Ni}$-EDTA chelates $(3,4)$. With the exception of the EDTA chelates, derivatization with the above compounds has been monitore by PAGE. However, for the radioisotopically labeled chelates, protein derivatization was monitored by scintillation counting $(3,4)$.

While PAGE and scintillation counting (above) provide direct assays for thiol derivatization, thiol derivatization can also be monitored indirectly for chemical reporters that cannot be assayed directly. This is done by assaying the unreacted thiols before and after derivatization with the primary reagent using a detectable (fluorescent) second reagent. Such methodology is customarily used to assay thiol derivatization with the "chemical nuclease" FeBABE [Iron (S)1-(p-bromoacetamidobenzyl)ethylenediaminetetraacetate (6)]. FeBABE is a chemical reporter similar to the above chelates, designed for assaying proteinnucleic acid proximity within proteinnucleic acid complexes. A disadvantage would be that un-reacted thiols may have become chemically modified during derivatization (e.g., thiol oxidation by the $\mathrm{Fe}^{3+}$ moiety of FeBABE when using the FeBABE reporter). Moreover, indirect measurement requires the use of a second reagent that reacts efficiently with thiols.

Mass spectrometry (MS) provides an opportunity to simultaneously detect both reacted and un-reacted thiols, provided the sample can be ionized. However, MS would be quantitative only to the extent that ionization is unaffected by covalent attachment of the reporter. Neither the "indirect", MS-based, nor scintillation assays would indicate, directly, whether derivatization with the primary reagent has inhibited the ability of the protein to form non-covalent (protein-protein or protein-nucleic acid) complexes. With the indirect assay, for example, the propensity of the second reagent to inhibit complex formation may be unrelated to that of the primary one, and with MS, maintaining the integrity of complexes during ionization can be challenging (if not impossible) to do. The direct detection of PAGE-fractionated complexes would seem preferable.

Therefore, we have extended the applicability of direct ${ }^{55} \mathrm{Fe}$ detection by implementing a PAGE ("in-gel") detection assay for protein derivatization with FeBABE. The protein to be assayed is first derivatized with the BABE portion of FeBABE, and then the mixture is incubated with ${ }^{55} \mathrm{FeCl}_{3}$. ${ }^{55} \mathrm{FeBABE}-$ labeled proteins are subjected to SDS-PAGE to examine the extent of derivatization; in parallel, an electrophoretic mobility shift assay (EMSA) gel can be run to assess complex formation by the derivatized pro-

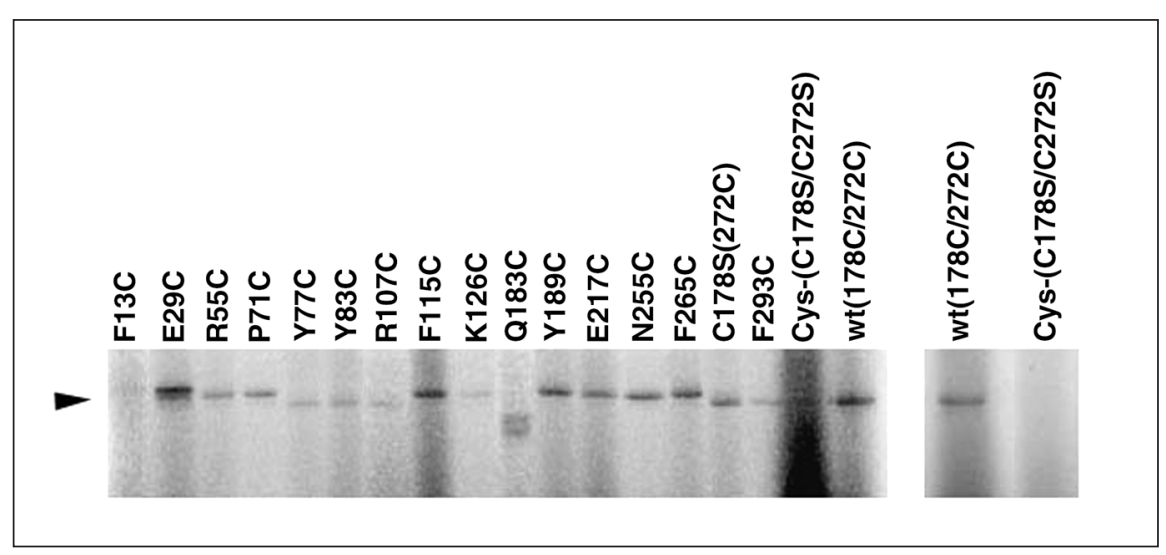

Figure 1. Thiol-FeBABE derivatization of protein samples. Main panel: demonstration of protein labeling with ${ }^{55} \mathrm{Fe}$. The storage phosphograms of SDS polyacrylamide gels show the derivatization of each of 16 VP39 single-cysteine proteins with ${ }^{55} \mathrm{FeBABE}$, along with wild-type VP39 (two cysteines, positions 178 and 272) and cysteine-minus VP39 control. Q183C preparations were dominated by two proteolytic products, as noted elsewhere (7). ${ }^{55} \mathrm{Fe}$ binding to VP39 was not detected in the absence of prior BABE coupling (data not shown). Right panel: To show clearly the absence of signal for cysteine-minus protein wild-type and cysteine-minus controls from another experiment (in which the amount of ${ }^{55} \mathrm{Fe}$ was equal to that used for the mutants in the main panel) are shown. Arrowhead indicates VP39-55FeBABE. 


\section{Proteomic Technologies}

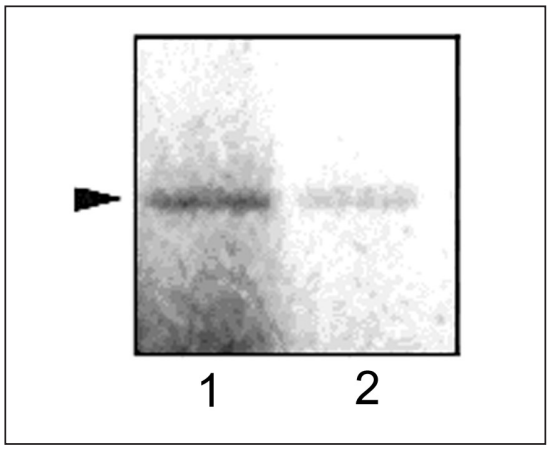

Figure 2. Chelation before coupling and coupling before chelation. The storage phosphogram shows an SDS polyacrylamide gel of the ${ }^{55} \mathrm{Fe}-1 \mathrm{a}-$ beled single cysteine mutant P71C. Lane 1 depicts $55 \mathrm{Fe}$ labeling exactly as described in Figure 1. In lane 2, the order of steps has been changed such that ${ }^{55} \mathrm{Fe}$ is first incubated with $\mathrm{BABE}$, after which the single cysteine of the protein is derivatized with the $55 \mathrm{FeBABE}$ complex. Other than the modified order of steps, all experimental conditions were identical. The lower level of labeling for experiments in which the chelate is formed before derivatization was observed for each of several single cysteine mutants tested (data not shown). Arrowhead indicates VP39_55FeBABE. teins. Being an Auger electron emitter, ${ }^{55} \mathrm{Fe}$ is readily detectable in the dried gels using either $\mathrm{X}$-ray film (neither intensifying screen nor fluorography being necessary) or a standard storagephosphor screen and electronic imager (such as the Bio-Rad FX; Bio-Rad Laboratories, Hercules, CA, USA).

Figure 1 shows SDS-PAGE data for a panel of 16 single-cysteine mutants of the 333 amino acid vaccinia virus protein VP39. The following experimental conditions were used in Figure 1. Before reaction with $\mathrm{BABE}$, proteins were at a concentration of $1 \mu \mathrm{M}$ in $10 \mathrm{mM}$ HEPES, pH 8.0, $25 \mathrm{mM} \mathrm{NaCl}, 0.1 \mathrm{mM}$ EDTA, $10 \%$ glycerol, $0.01 \%$ Nonidet $^{\mathrm{TM}}$ P-40. Seventeen picomoles of protein $\mathrm{F} 13 \mathrm{C}$ or $35.5 \mathrm{pmol}$ each of the remaining VP39 proteins $(7,9)$ were mixed with $1 \mu \mathrm{L}$ BABE (Dojindo Molecular Technologies, Gaithersburg MD, USA; $17.75 \mathrm{mM}$ in DMSO), and the mixtures were incubated at room temperature for
4 h. ${ }^{55} \mathrm{FeCl}_{3}[(18.7 \mu \mathrm{L}) 20 \mathrm{mCi} / \mathrm{mL}$ in $0.5 \mathrm{M} \mathrm{HCl}$; PE-NEN] was dried under vacuum and then re-dissolved in DMSO to a final concentration of 1.43 $\mathrm{mM}$. One microliter of this (sufficient to render approximately $10 \%$ saturation of $\mathrm{BABE}$ with ${ }^{55} \mathrm{Fe}^{3+}$ ) was added to each of the protein-BABE conjugation mixtures, except for the cys-minus protein. A greater amount $(5 \mu \mathrm{L})$ was added to the latter in an attempt to check for possible background labeling of the cysteine-minus control, resulting in a high background throughout this gel lane. After $60 \mathrm{~min}$ incubation at room temperature, $18 \mu \mathrm{L}$ were withdrawn from each reaction mixture, mixed with $6 \mu \mathrm{L} 4 \times$ SDS-PAGE loading buffer, and loaded on a $10 \%$ SDS polyacrylamide gel. After electrophoresis ( $3 \mathrm{~h}$ at $25 \mathrm{~mA}$ ), the gel was dried and imaged on storage phosphor using a Bio-Rad FX Imager.

Although a signal could be detected 
for each mutant (even for mutant F13C and for the full-length band in the preparation of Q183C after prolonged exposure), significant variability in signal strength was observed among the 16 mutants, indicating variability between the thiols in coupling efficiency. Changing the order of steps such that the chelate is formed before thiol derivatization yielded protein that was ${ }^{55} \mathrm{Fe}$-labeled almost as abundantly as that in which coupling preceded chelation (Figure 2).

Upon subjecting aliquots of the 16 derivatized proteins shown in Figure 1 to the EMSA (1) by incubation of the derivatized proteins with vaccinia protein VP55 plus an appropriate oligonucleotide followed by nondenaturing gel electrophoresis (excluding EDTA from the EMSA gels), the ${ }^{55} \mathrm{FeBABE}-\mathrm{cou}-$ pled proteins were observed to be excluded from EMSA complexes (data not shown). In control experiments in which aliquots of the ${ }^{55} \mathrm{FeBABE}-\mathrm{de}-$ rivatized VP39 mutant or the oligonucleotide were ${ }^{32} \mathrm{P}$-end-labeled immediately before the EMSA, complexes were readily detected (data not shown). Apparently, complex formation was severely impaired by prior FeBABE coupling to each mutant protein.

Although the in-gel detection assay provides a convenient way to assess the propensity of the derivatized protein to form non-covalent complexes (above), absolute quantitation of EDTA:protein stoichiometry might be problematic with respect to, say, the previously described scintillation counting methods $(3,4)$ because of quenching by the gel slice. However, relative quantitation might be possible with the in-gel method, by co-electrophoresing a standard with rigorously known EDTA:protein stoichiometry.

\section{ACKNOWLEDGMENTS}

We thank Dr. Claude Meares for the kind gift of FeBABE, advice on its use, and the suggestion of a commercial supplier of unchelated BABE, Dr. Akihiro Oguro for the mutant proteins, and an anonymous referee for comments. This work was supported by National Institutes of Health grant no. R01 GM501953.

\section{REFERENCES}

1.Deng, L., L. Beigelman, J. Matulic-Adamic, A. Karpeisky, and P.D. Gershon. 1997. Specific recognition of an $\mathrm{rU}_{2}-\mathrm{N}_{15}$-rU motif by VP55, the vaccinia virus poly $(\mathrm{A})$ polymerase catalytic subunit. J. Biol. Chem. 272:3154231552 .

2.Ebright, Y.W., Y. Chen, Y. Kim, and R.H. Ebright. 1996. S-[2-(4-azidosalicylamido) ethylthio]-2-thiopyridine: radioiodinatable, cleavable, photoactivatable cross-linking agent. Bioconjug. Chem. 7:380-384.

3.Ebright, Y.W., Y. Chen, R.D. Ludescher, and R.H. Ebright. 1993. $N$-(iodoacetyl)- $p$ phenylenedimine-EDTA: areagent for highefficiency incorporation of an EDTA-metal complex at a rationally selected site within a protein. Bioconjug. Chem. 4:219-225.

4.Ebright, Y.W., Y. Chen, P.S. Pendergrast, and R.H. Ebright. 1992. Incorporation of an EDTA-metal complex at a rationally selected site within a protein: application to EDTAiron DNA affinity cleaving with catabolite gene activator protein (CAP) and Cro. Biochemistry 31:10664-10670.

5.Ercal, N., P. Yang, and N. Aykin. 2001. Determination of biological thiols by high-performance liquid chromatography following derivatization by ThioGlo maleimide reagents. J. Chromatogr. B Biomed. Sci. Appl. 753:287-292.

6.Greiner, D.P., R. Miyake, J.K. Moran, A.D. Jones, T. Negishi, A. Ishihama, and C.F. Meares. 1997. Synthesis of the protein cutting reagent iron (S)-1-(p-bromoacetamidobenzyl) ethylenediaminetetraacetate and conjugation to cysteine side chains. Bioconjug. Chem. $8: 44-48$.

7.Oguro, A., L. Johnson, and P.D. Gershon. 2002. Path of an RNA ligand around the surface of the vaccinia VP39 subunit of its cognate VP39-VP55 protein heterodimer. Chem. Biol. 9:679-690.

8.Parvari, R., I. Pecht, and H. Soreq. 1983. A microfluorometric assay for cholinesterases, suitable for multiple kinetic determinations of picomoles of released thiocholine. Anal. Biochem. 133:450-456.

9.Shi, X., T.G. Bernhardt, S.-M. Wang, and P.D. Gershon. 1997. The surface region of the bifunctional vaccinia RNA modifying protein VP39 that interfaces with poly(A) polymerase is remote from the RNA binding cleft used for its mRNA 5' cap methylation function. J. Biol. Chem. 272:23292-23302.

Received 12 December 2001; accepted 10 July 2002.

\section{Address correspondence to:}

Dr. Paul D. Gershon

Institute of Biosciences and Technology

Texas A\&M University System Health Science

Center

Houston, TX 77030, USA

e-mail:pgershon@ibt.tamu.edu 